\title{
Decision-Support Tool in Prehospital Care: A Systematic Review of Randomized Trials
}

\author{
Magnus Hagiwara, RN, MScN; ${ }^{1}$ Maria Henricson, RN, CCRN, PhD; ${ }^{2}$ Anders Jonsson, PhD; ${ }^{3}$ \\ Björn-Ove Suserud, $\mathrm{PhD}^{1}$
}

1. University of Borås, School of Health Sciences, Borås, Sweden

2. Assistant Professor, Jönköping University, School of Health Sciences, Jönköping

3. Borås University, School of Health Sciences; Associate Professor, Swedish Armed Forces, Centre for Defence Medicine, Sweden

\author{
Correspondence: \\ Magnus Hagiwara \\ University of Borås \\ School of Health Sciences \\ 50190 Borås \\ Sweden \\ E-mail: magnus.hagiwara@hb.se
}

Keywords: Prehospital decision support tool, prehospital care, emergency medical services, emergency medical technician

\begin{abstract}
Abbreviations:
DST: Decision Support Tool

ECP: Emergency Clinical Pathway

EMS: Emergency Medical Services

RCT: Randomized Controlled Trial
\end{abstract}

Received: 15 July 2010

Accepted: 09 August 2010

Revised: 23 August 2010

doi:10.1017/S1049023X11006534

\begin{abstract}
Objective: The objective of this study was to evaluate the effects of the decision support tool (DST) on the assessment of the acutely ill or injured out-of-hospital patient.

Methods: This study included systematic reviews of randomized controlled trials (RCT) where the DST was compared to usual care in and out of the hospital setting. The databases scanned include: (1) Cochrane Reviews (up to January 2010); (2) Cochrane Controlled Clinical Trials (1979 to January 2010); (3) Cinahl (1986 to January 2010); and (4) Pubmed/ Medline (1926 to January 2010). In addition, information was gathered from related magazines, prehospital home pages, databases for theses, conferences, grey literature and ongoing trials.

Results: Use of the DST in prehospital care may have the possibility to decrease "time to definitive care" and improve diagnostic accuracy among prehospital personnel, but more studies are needed.

Conclusions: The amount of data in this review is too small to be able to draw any reliable conclusions about the impact of the use of the DST on prehospital care. The research in this review indicates that there are very few RCTs that evaluate the use of the DST in prehospital care.
\end{abstract}

Hagiwara M, Henricson M, Jonsson A, Suserud BO: Decision-support tool in prehospital care: A systematic review of randomized trials. Prehosp Disaster Med 2011;26(4):1-11.

\section{Introduction}

Prehospital patient assessment is different compared to assessments provided in hospitals. Often, the ambulance personnel are sent to medical emergencies with very little background information. The person who initiates the call for help sometimes is not able to describe the situation, and therefore, the ambulance personnel have difficulties preparing themselves for the task. The ambulance personnel's work situation has been described as "extreme". "They have routinely required working in environments of extreme and con-

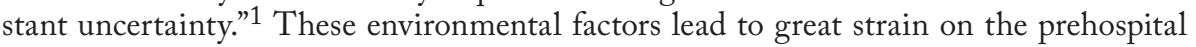
caregiver's ability when assessing patients. In addition, there have been very few randomized controlled trials (RTC) that have evaluated prehospital care. ${ }^{2}$ When 14 ambulance nurses in Sweden were asked about factors that influence their decision-making, several factors that were crucial for the decision were elicited. Important factors included: (1) time of day; (2) season and weather; (3) amount of time before other resources could arrive to the scene; (4) if there was threat of security; (5) stress; (6) environment; (7) diagnosis and; (8) type of patient assessed. ${ }^{3}$ A qualitative study by Wireklint Sundström described the need of an "open mind" in the performance of prehospital assessments, and noted that prehospital caregivers must put their preconceived ideas aside when they meet and assess patients. ${ }^{4} \mathrm{As}$ assessments and treatments of patients in prehospital emergency medical care are becoming more advanced and complex, prehospital emergency care is on the eve of a new and emerging role. ${ }^{5}$ Many organizations are searching for alternatives to Accident and Emergency (A\&E) treatments, ${ }^{6}$ but according to a recent systematic review, prehospital personnel's preparedness for this task is poor. ${ }^{7}$ Another future task will include "clinical pathways". Clinical pathways help to reduce the number and severity of in-hospital complications. ${ }^{8}$ If the prehospital care becomes a part of clinical pathways, it will impose many demands on prehospital personnel to make correct assessments. 
This indicates that decision support tools (DST) probably will be required in the future. A decision-support tool often is defined as electronic or non-electronic active knowledge systems that use at least two objects of patient data to generate case-specific advice designed to support clinical decision-making. Decision support can be defined as protocols, guidelines, and different triage systems. The function of the decision-support data is to create individual patient assessments or recommendations that support all level of prehospital medical personnel.

Using a Delphi consultation, the question, "What are the highest priorities for research in prehospital care?" was studies, with one of the research issues being the DST. ${ }^{9}$ Several systematic reviews have evaluated the efficacy of the use of the DST in primary health care and in hospital settings, ${ }^{10-14}$ which demonstrated that use of DSTs can improve the performance of health workers, but its effects on patient outcomes still was unclear. There has been no published review that has evaluated effects of the use of the DST in prehospital emergency care. The aim of this systematic review is to investigate the DST's impact on prehospital patient assessment.

\section{Methods}

Selection Criteria

Type of Studies-Randomized controlled trials that compared the use of a DST with usual care were included in this systematic review. Included studies may have used patients, prehospital personnel, hospitals, or ambulance stations/organizations as the unit of randomization.

Evidence of interventional studies depends on whether the study was randomized or not. Studies have suggested that non-randomized, controlled trials are associated with selection bias, and should not be included in systematic reviews of interventions. ${ }^{15}$

In this review, the SIGN50 checklist was used as a rating tool for quality assessment of included studies. ${ }^{16}$ In a study that evaluated different kinds of quality assessment instruments, the conclusion was that this checklist is the best for evaluating assessments of randomized, controlled trials. ${ }^{17}$ To be included in the review, a study must be rated as a “+” or “++". To be rated as "++", the studies must have met all or most of the criteria, and the conclusion from the study was unlikely to change. The rating "+" indicated that some of the criteria are fulfilled, but those criteria not fulfilled were unlikely to have altered the conclusion.

Types of Participants - Studies in which prehospital personnel assess patients with an acute illness or injuries in and out of the hospital setting are eligible.

Types of Interventions-Decision support can be both electronic and non-electronic. One definition of an electronic DST is, "active knowledge systems which use two or more items of patient data to generate case-specific advice". ${ }^{18}$ Perreault and Metzgerlist used four key functions listed below: ${ }^{19}$

1. Administrative-Supporting clinical coding and documentation, authorization of procedures, and referrals;

2. Managing Clinical Complexity and Details-Keeping patients on research and chemotherapy protocols; tracking orders, follow-up of referrals, and preventive care;

3. Cost Control-Monitoring medication orders; avoiding duplicate or unnecessary tests; and
4. Decision Support-Supporting clinical diagnosis and treatment plan processes, and promoting use of best practices, condition-specific guidelines, and population-based management."

To be eligible for this review, the studies had to fulfill criteria \#4.

For this review, the inclusion criteria were studies comparing the use of an electronic or non-electronic DST (where patient-specific assessments or recommendations were given to ambulance personnel) with usual care in a clinical setting. Decision support can be protocols, guidelines, triage systems, and computer based DSTs. Triage systems without decision support were excluded. For example, if a triage system only gave the patient a color, number, or letter to use in the emergency department to prioritize the patient, it did not qualify as a DST. If the triage system also gave the care provider in the ambulance a suggestion of further examination and treatment, it could be qualified as a DST. In cases in which it was not possible to determine if the DST or the interventions (i.e., medical treatment or education), had influenced the outcome, the study was excluded.

\section{Types of Outcome Measures}

Primary Outcomes

Diagnostic Accuracy - Can the use of a DST improve the ambulance personnel's ability to correctly diagnose a prehospital patient? Diagnostic ability is important to give the patient sufficient treatment and transport to which he/she had the right level of care. Studies that evaluated a DST's impact on diagnostic ability were eligible.

Compliance with Recommended Practice-Compliance with guidelines has importance to patient outcome. ${ }^{20}$ This review attempted to establish if a DST increased the ambulance personnel's adjustment to guidelines.

Time to Definitive Care-Many patients may have been objects of "clinical pathways" or "fast tracks" when they are transported to different types of treatment centrals. Examples of definitive care include emergency rooms, stroke units (SUs), primary percutaneous coronary intervention (PCI) centers, or trauma centers. This review includes studies that evaluated the impact of the DST on the time from call to definitive care.

Patient Outcomes-Studies that compared the impact of the DST with usual care on the patient's outcome were included. Patient outcome may have been measures of morbidity or mortality.

\section{Secondary Outcomes}

1. Patient Satisfaction-Studies that evaluated the patient's satisfaction with the assessments and treatments with a DST were eligible; and

2. Personnel Satisfaction: Studies that evaluated the satisfaction of prehospital personnel with DSTs were eligible.

\section{Search Methods}

Electronic Searches-Searches were performed in the following databases from January until April 2010: (1) Cochrane Reviews (up to January 2010); (2) Cochrane Controlled Clinical Trials (1979 to January 2010); (3) Cinahl (1986 to January 2010); and (4) Pubmed/Medline (1926 to January 2010). There was no restriction of language or year of publication. The search 
strategy was pilot tested and adjusted before performance of the final search. The combination of the following terms was used in the search:

1. MeSh Terms: emergency medical services, emergency medical technicians, emergency treatment, emergency medicine, ambulances, air ambulances, first aid, military medicine, emergency medical service communication systems, decision support systems (clinical), decision making (clinical), decision support techniques, decision making, computerized patient record, decision making (patient), clinical protocols, and emergency treatment.

2. Text Terms: prehospital, pre-hospital, paramedic, ambulance, out-of-hospital, out of hospital, EMS, EMT, emergency services, emergency medical service, emergency technician, emergency practitioner, emergency dispatch, first responder, emergency rescue, emergency triage, patient assessment.

Searching Other Resources-In addition to searching medical databases, a hand search was performed in 18 relevant magazines: Emergency Medicine Journal (March 1984 to January 2010), Emergency Nurse (February 2002 to December 2009), Medical Informatics $\boldsymbol{E}^{\circ}$ the Internet in Medicine (January 1999 to December 2009), Military Medicine (January 2003 to January 2010), Medical Decision Making (January 1981 to January 2010), BMC Medical Informatics And Decision Making (January 2001 to January 2010), Journal of Telemedicine and Telecare (January 1996 to January 2010) Air Medical Journal (January 1993 to January 2010), Australian Journal of Emergency Management (January 1998 to January 2010), Canadian Journal of Emergency Medicine (January 1999 to January 2010), Emergency Medicine Australasia (January 1999 to 2010), Journal of Emergency Primary Health Care (January 2003 to January 2010), Emergency Medical Services Magazine (January 2003 to January 2010), Journal of Air Medical Transport (January 1989 to January 1992), Prehospital and Disaster Medicine (January 2002 to January 2009), Prehospital Emergency Care (January 1997 to January 2010), Pre-Hospital Immediate Care (January 2000 to December 2000), IEEE Transactions on information Technology in Biomedicine (January 1997 to January 2010).

Nine prehospital home pages were searched: Australian Directory of Prehospital Research (February 2010), National Association of EMS Physicians (February 2010), National Association of EMS Educators (February 2010), AMBEX (UK) (February 2010), Australasian College of Ambulance (February 2010), World Congress on Disaster and Emergency Medicine (February 2010), Society for Academic Emergency Medicine (February 2010), Canadian Association of Emergency Physicians (February 2010), American College of Emergency Physicians (ACEP) Meeting (February 2010).

In addition, searches were conducted in two databases for conference abstracts: Biological Abstracts/RRM (February 2010), British Library Inside (February 2010), fifteen databases for present clinical trials: The Australian New Zealand Clinical Trials Registry (February 2010), CenterWatch Clinical Trials Listing Service (February 2010), ClinicalTrials.gov register (February 2010), Community Research E Development Information Service (of the European Union) (trials and other research) (February 2010), Current Controlled Trials metaRegister of Controlled Trials (mRCT) - active registers (February 2010), European Medicines
Agency (February 2010), WHO International Clinical Trials Registry (February 2010), Hong Kong clinical trials register (February 2010), Indian clinical trials registry - Clinical Trials Registry-India (February 2010), Netherlands trial register (February 2010), South African National Clinical Trial Register (February 2010), UK Clinical Research Network Portfolio Database (February 2010), University hospital Medical Information Network (UMIN) Clinical Trials Registry (for Japan) - UMIN CTR (February 2010), three databases for grey literature: Open single database (up to January 2010), The National Technical Information Sevice database (1962 to January 2010), PsykEXTRA database (up to January 2010) and three databases for theses: Dissertations $\mathcal{F}^{\circ}$ Theses (1975 to January 2010), DIVA (up to January 2010), Index to Theses (up to January 2010). The reference list of relevant studies was also searched.

Selection of Studies - In the first step, one investigator screened titles and abstracts for eligible studies. Abstracts of interest were downloaded to a reference manager and full text copies were retrieved. In the second step, full text articles were assessed for eligibility by two independent investigators. Studies that did not fulfil the inclusion criteria were excluded. In the last step, the SIGN 50 checklists for RCTs was used for methodological quality assessment. Disagreement on screening results was resolved by discussion. Data was extracted and handled by a data abstraction form specially developed for this review.

\section{Results}

\section{Results of the Search}

The database search initially identified 5,929 citations. After excluding studies in the first screening and removing duplicates, 59 citations remained. The search in magazines, conference abstracts, ongoing studies, home pages, theses and reference lists provided an additional 14 citations. Full text screening was performed for 73 articles. Of those, 33 studies were eligible for final screening, and 31 studies were excluded because of study design (Figure 1). One ongoing trial of the prehospital application of DST was found (Snooks, TRIAL REGISTRATION: ISRCTN10538608.), ${ }^{20}$ but no data were available at the time of writing. Two trials fulfilled the inclusion criteria. ${ }^{21,22}$ Due to differences in the types of statistical methods reported in the trials, a meta-analysis was not possible. Instead, the data were reported in tabular and narrative format.

\section{Excluded Studies}

Among the excluded studies (Table 1) included in this review were several interesting cohort studies. There were three studies that compared the intervention with a prospective control group. ${ }^{23-25}$ They all demonstrated a positive result that favored the intervention, but the risk of bias was large. If the intervention group consisted of volunteers who were compared to non-volunteers in the control group, it was difficult to establish which factors influenced the outcome due to the chance of selection bias. There could have been a risk that the volunteer group consisted of more interested and qualified personnel, and this may have influenced the results. In eight other studies, ${ }^{26-33}$ the intervention was compared with a retrospective control group. They all also demonstrated a significant favor toward the intervention group, but it is difficult to determine if the two compared groups were homogeneous enough to draw any conclusions. 


\section{Flow chart}

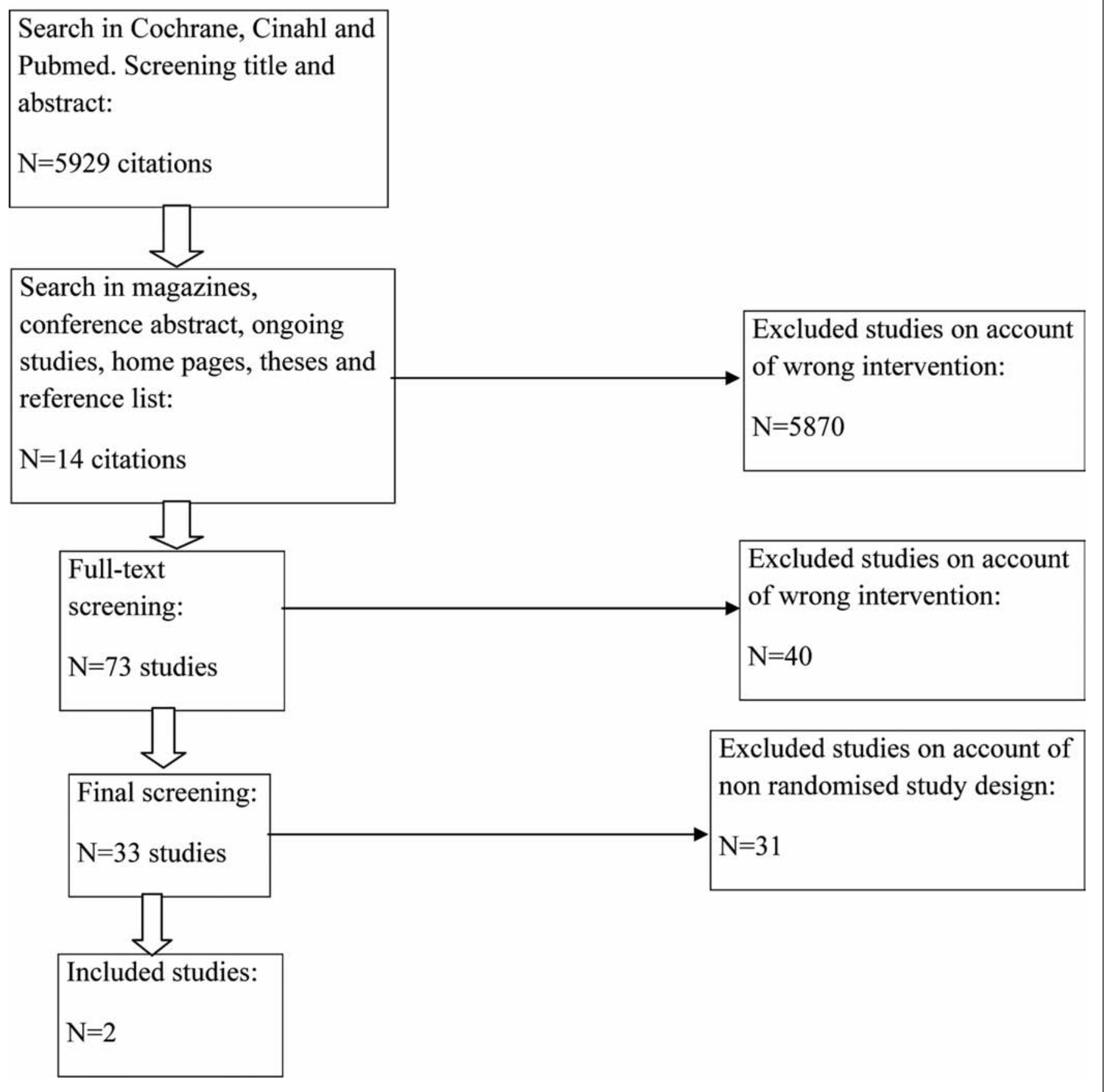

Figure 1-Flow diagram of included and excluded studies

Hagiwara @ 2011 Prehospital and Disaster Medicine

\section{Effects of DST}

Diagnostic Accuracy - There was one eligible study (Table 2) that presented data that described the DST impact on diagnostic accuracy. ${ }^{21}$ The aim of the trial was to assess the impact of an Emergency Clinical Pathway (ECP) for acute stroke patients. The study involved both emergency medical services (EMS) and emergency departments. The EMS group used the Cincinnati prehospital Stroke Scale (CSS) as a DST. This review uses data from the EMS group. The number of eligible patients being appropriately referred to the stroke unit (SU) by the EMS group was an outcome. In the intention to treat analysis there was a statistically significant favor for the intervention group. Of 109 eligible patients 68 patients $(62.4 \%)$ were referred to the SU compared to 9 of 56 (16.1\%) eligible patients in the control group $(\mathrm{RR}=4.09 ; 95 \% \mathrm{CI}=1.84$ to 5.59$)$. The study also provided data of how many eligible stroke patients were receiving thrombolysis at the SU. In the intervention group 8 of 44 (18.2\%) were receiving thrombolysis, and in the control group 0 of $22(0 \%)$ were 


\begin{tabular}{|c|c|c|c|c|c|c|}
\hline Study & Design & $\begin{array}{l}\text { Reason for } \\
\text { exclusion }\end{array}$ & Intervention & $\begin{array}{l}\text { Number of } \\
\text { participants }\end{array}$ & Outcomes & Results \\
\hline $\begin{array}{l}\text { Bray } \\
2005^{23}\end{array}$ & $\begin{array}{l}\text { Non randomised } \\
\text { cohort study. }\end{array}$ & $\begin{array}{l}\text { Study } \\
\text { design. }\end{array}$ & $\begin{array}{l}\text { Prehospital stroke tool } \\
\text { (FAST). }\end{array}$ & $\begin{array}{l}\text { Intervention: } \\
\mathrm{n}=11 \\
\text { Control: } \\
\mathrm{n}=43 \\
\text { (patients) }\end{array}$ & $\begin{array}{l}\text { Diagnostic } \\
\text { accuracy. }\end{array}$ & $\begin{array}{l}\text { The sensitivity for the } \\
\text { intervention group in } \\
\text { identifying stroke improved } \\
\text { from } 78 \%(95 \% \text { confidence } \\
\text { interval }[\mathrm{Cl}]=63 \% \text { to } 88 \%) \\
\text { to } 94 \%(95 \% \mathrm{Cl}=86 \% \text { to } \\
98 \%)(p=0.006) \text {. There } \\
\text { was no change in stroke } \\
\text { diagnosis for the control } \\
\text { group } 78 \%(95 \% \mathrm{Cl}= \\
71 \% \text { to } 84 \%) \text { to } 80 \% \\
(95 \% \mathrm{Cl}=72 \% \text { to } 87 \%) \\
(p=0.695) \text {. }\end{array}$ \\
\hline $\begin{array}{l}\text { Burton } \\
2005\end{array}$ & $\begin{array}{l}\text { Prospective } \\
\text { observational } \\
\text { study. }\end{array}$ & $\begin{array}{l}\text { Study } \\
\text { design. }\end{array}$ & $\begin{array}{l}\text { Spine-assessment } \\
\text { protocol. }\end{array}$ & $\begin{array}{l}\mathrm{n}=2,220 \\
\text { (patients) }\end{array}$ & $\begin{array}{l}\text { Diagnostic } \\
\text { accuracy. }\end{array}$ & $\begin{array}{l}\text { Decision "not to } \\
\text { immobilize" increased } \\
\text { with } 40 \% \text { of EMS } \\
\text { trauma patients. }\end{array}$ \\
\hline $\begin{array}{l}\text { Cayten } 24 \\
1983\end{array}$ & $\begin{array}{l}\text { Non-randomized } \\
\text { cohort study. }\end{array}$ & $\begin{array}{l}\text { Study } \\
\text { design. }\end{array}$ & $\begin{array}{l}\text { Algorithm for } \\
\text { prehospital assessment } \\
\text { of arrhythmias. }\end{array}$ & $\begin{array}{l}\text { Intervention: } \\
\mathrm{n}=73 \\
\text { Control: } \\
\mathrm{n}=65 \\
\text { (patients) }\end{array}$ & $\begin{array}{l}\text { Patient } \\
\text { outcomes. }\end{array}$ & $\begin{array}{l}\text { The intervention group } \\
\text { improved the rates at } \\
\text { which their patients with } \\
\text { ventricular fibrillation } \\
\text { survived to hospital from } \\
11.25 \text { to } 15.1 \%(p=0.02) \text {. } \\
\text { In the control group the } \\
\text { survival decreased. }\end{array}$ \\
\hline $\begin{array}{l}\text { Chelsea } \\
1998^{35}\end{array}$ & $\begin{array}{l}\text { Retrospective } \\
\text { study. }\end{array}$ & $\begin{array}{l}\text { Study } \\
\text { design. }\end{array}$ & $\begin{array}{l}\text { Prehospital stroke } \\
\text { tool (Los Angeles } \\
\text { Prehospital Stroke } \\
\text { Screen). }\end{array}$ & $\begin{array}{l}\mathrm{n}=48 \\
\text { (patients) }\end{array}$ & $\begin{array}{l}\text { Diagnostic } \\
\text { accuracy. }\end{array}$ & $\begin{array}{l}\text { Of the } 48 \text { total stroke } \\
\text { patients (ischemic } \\
\text { plus hemorrhagic), } \\
44 \text { were correctly } \\
\text { identified by the LAPSS } \\
\text { ( } 92 \% \text { sensitivity). }\end{array}$ \\
\hline $\begin{array}{l}\text { Dhruva } \\
2007^{27}\end{array}$ & $\begin{array}{l}\text { Prospective, } \\
\text { observational } \\
\text { study. Before } \\
\text { and after. }\end{array}$ & $\begin{array}{l}\text { Study } \\
\text { design. }\end{array}$ & $\begin{array}{l}\text { Wireless } \\
\text { electrocardiogram } \\
\text { transmissions from the } \\
\text { field to the emergency } \\
\text { department. }\end{array}$ & $\begin{array}{l}\text { Intervention: } \\
\mathrm{n}=20 \\
\text { Retrospective } \\
\text { control group: } \\
\mathrm{n}=29\end{array}$ & $\begin{array}{l}\text { Time to } \\
\text { definitive } \\
\text { care. }\end{array}$ & $\begin{array}{l}\text { Mean door-to-cardiologist } \\
\text { notification time was } \\
\text { reduced by } 76 \mathrm{~min} \\
(61.4 \text { to }-14.6 \mathrm{~min} \text {, } \\
p=0.001) . \text { The door to } \\
\text { intervention time was } \\
\text { reduced by } 66 \mathrm{~min} \\
(145.6 \text { to } 80.1 \mathrm{~min} \text {, } \\
p=0.001) .\end{array}$ \\
\hline $\begin{array}{l}\text { Dorraine } \\
2004^{28}\end{array}$ & $\begin{array}{l}\text { Prospective, } \\
\text { observational } \\
\text { study. Before } \\
\text { and after. }\end{array}$ & $\begin{array}{l}\text { Study } \\
\text { design. }\end{array}$ & $\begin{array}{l}\text { Prehospital brain injury } \\
\text { guidelines. }\end{array}$ & $\begin{array}{l}\text { Intervention: } \\
\mathrm{n}=52 \\
\text { Retrospective } \\
\text { control group: } \\
\mathrm{n}=53 \\
\text { (patients) }\end{array}$ & $\begin{array}{l}\text { Patient } \\
\text { outcomes. }\end{array}$ & $\begin{array}{l}\text { Mortality at } 30 \text { days } \\
\text { reduced after } \\
\text { implementation from } \\
34.6 \% \text { to } 17.0 \% \\
(p=0.039) .\end{array}$ \\
\hline
\end{tabular}

Table 1-Study characteristics of excluded studies of prehospital decision support (continues)

Hagiwara @ 2011 Prehospital and Disaster Medicine 


\begin{tabular}{|c|c|c|c|c|c|c|}
\hline $\begin{array}{l}\text { Fries } \\
1994^{36}\end{array}$ & $\begin{array}{l}\text { Prospective, } \\
\text { observational } \\
\text { study. }\end{array}$ & $\begin{array}{l}\text { Study } \\
\text { design. }\end{array}$ & $\begin{array}{l}\text { Trauma Triage Rule in } \\
\text { prehospital trauma } \\
\text { triage (TTR). }\end{array}$ & $\begin{array}{l}\mathrm{n}=653 \\
\text { (patients) }\end{array}$ & $\begin{array}{l}\text { Compliance } \\
\text { with } \\
\text { recommended } \\
\text { practice }\end{array}$ & $\begin{array}{l}\text { Paramedic judgment had } \\
\text { a sensitivity of } \\
91 \% \text { and a specificity of } \\
60 \% \text {. Combining the TTR } \\
\text { and paramedic judgment } \\
\text { achieved a sensitivity of } \\
100 \% \text { and a specificity } \\
\text { of } 75 \% \text {. }\end{array}$ \\
\hline $\begin{array}{l}\text { Kidwell }{ }^{40} \\
2000\end{array}$ & $\begin{array}{l}\text { Prospective, } \\
\text { observational } \\
\text { study. }\end{array}$ & $\begin{array}{l}\text { Study } \\
\text { design. }\end{array}$ & $\begin{array}{l}\text { Los Angeles } \\
\text { Prehospital } \\
\text { Stroke Screen } \\
\text { (LAPSS) }\end{array}$ & $\begin{array}{l}\mathrm{n}=1,298 \\
\text { (patients) }\end{array}$ & $\begin{array}{l}\text { Diagnostic } \\
\text { accuracy. }\end{array}$ & $\begin{array}{l}\text { Positive predictive value } \\
\text { increased to } 97 \% \\
(95 \% \mathrm{Cl}=84 \% \text { to } 99 \%) \text {. }\end{array}$ \\
\hline $\begin{array}{l}\text { Klein } \\
\text { 1997[41] }\end{array}$ & $\begin{array}{l}\text { Retrospective } \\
\text { observational } \\
\text { study. }\end{array}$ & $\begin{array}{l}\text { Study } \\
\text { design. }\end{array}$ & $\begin{array}{l}\text { On-line medical control } \\
\text { (OLMC). }\end{array}$ & $\begin{array}{l}\mathrm{n}=774 \\
\text { (patients) }\end{array}$ & $\begin{array}{l}\text { Compliance } \\
\text { with } \\
\text { recommended } \\
\text { practice. }\end{array}$ & $\begin{array}{l}\text { OLMC does not improve } \\
\text { adherence to protocol } \\
\text { or the quality of care } \\
\text { provided in the treatment } \\
\text { of atraumatic illness. }\end{array}$ \\
\hline $\begin{array}{l}\text { Knapp } \\
2009^{42}\end{array}$ & $\begin{array}{l}\text { Prospective, } \\
\text { observational } \\
\text { study. }\end{array}$ & $\begin{array}{l}\text { Study } \\
\text { design. }\end{array}$ & $\begin{array}{l}\text { Non-transport } \\
\text { guidelines. }\end{array}$ & $\begin{array}{l}\mathrm{n}=104 \\
\text { (patients) }\end{array}$ & $\begin{array}{l}\text { Compliance } \\
\text { with } \\
\text { recommended } \\
\text { practice. }\end{array}$ & $\begin{array}{l}\text { EMS providers } \\
\text { underestimate the } \\
\text { potential severity of } \\
\text { illness. }\end{array}$ \\
\hline $\begin{array}{l}\text { Kothari } \\
1999^{43}\end{array}$ & $\begin{array}{l}\text { Prospective, } \\
\text { observational } \\
\text { study. }\end{array}$ & $\begin{array}{l}\text { Study } \\
\text { design. }\end{array}$ & $\begin{array}{l}\text { The Cincinnati } \\
\text { Prehospital Stroke } \\
\text { Scale } \\
\text { (CPSS). }\end{array}$ & $\begin{array}{l}\mathrm{n}=171 \\
\text { (patients) }\end{array}$ & $\begin{array}{l}\text { Diagnostic } \\
\text { accuracy. }\end{array}$ & $\begin{array}{l}\text { The CPSS has excellent } \\
\text { reproducibility among } \\
\text { prehospital personnel and } \\
\text { physicians. }\end{array}$ \\
\hline
\end{tabular}

Table 1 (continued) - Study characteristics of excluded studies of prehospital decision support 


\begin{tabular}{|c|c|c|c|c|c|c|}
\hline $\begin{array}{l}\text { Le May } \\
2006^{30}\end{array}$ & $\begin{array}{l}\text { Prospective, } \\
\text { observational } \\
\text { study. Before and } \\
\text { after. }\end{array}$ & $\begin{array}{l}\text { Study } \\
\text { design. }\end{array}$ & $\begin{array}{l}\text { ECG telemedicin, } \\
\text { PCl protocol. }\end{array}$ & $\begin{array}{l}\text { Intervention: } \\
\mathrm{n}=108 \\
\text { Retrospective } \\
\text { control: } \\
\mathrm{n}=225\end{array}$ & $\begin{array}{l}\text { Patient } \\
\text { outcomes. }\end{array}$ & $\begin{array}{l}\text { In-hospital mortality } \\
\text { was } 1.9 \% \text { in the } \\
\text { paramedic-referred } \\
\text { primary PCl group versus } \\
8.9 \% \text { in the control group } \\
(p=0.017) .\end{array}$ \\
\hline $\begin{array}{l}\text { Meisel } \\
2009^{44}\end{array}$ & $\begin{array}{l}\text { Retrospective } \\
\text { observational } \\
\text { study. }\end{array}$ & $\begin{array}{l}\text { Study } \\
\text { design. }\end{array}$ & $\begin{array}{l}\text { Scoring system to } \\
\text { predict hospital } \\
\text { admission versus } \\
\text { ED discharge for } \\
\text { patients transported by } \\
\text { ambulance. }\end{array}$ & $\begin{array}{l}\mathrm{n}=1102 \\
\text { (patients) }\end{array}$ & $\begin{array}{l}\text { Compliance } \\
\text { with } \\
\text { recommended } \\
\text { practice. }\end{array}$ & $\begin{array}{l}\text { The ability of this EMS } \\
\text { rule to predict the } \\
\text { likelihood of hospital } \\
\text { admission appears valid. }\end{array}$ \\
\hline $\begin{array}{l}\text { Muhr } \\
1999^{31}\end{array}$ & $\begin{array}{l}\text { Prospective, } \\
\text { observational } \\
\text { study. Before and } \\
\text { after }\end{array}$ & $\begin{array}{l}\text { Study } \\
\text { design }\end{array}$ & $\begin{array}{l}\text { Spinal clearance } \\
\text { algorithm }\end{array}$ & $\begin{array}{l}\text { Intervention: } \\
\mathrm{n}=281 \\
\text { Retrospective } \\
\text { control group: } \\
\mathrm{n}=293\end{array}$ & $\begin{array}{l}\text { Compliance } \\
\text { with } \\
\text { recommended } \\
\text { practice }\end{array}$ & $\begin{array}{l}33 \% \text { reduction in utilization } \\
\text { of spinal immobilisation } \\
(95 \% \mathrm{Cl}=27.2 \%-38.8 \%) \text {. }\end{array}$ \\
\hline $\begin{array}{l}\text { Norcross } \\
1995^{46}\end{array}$ & $\begin{array}{l}\text { Prospective, } \\
\text { observational } \\
\text { study. }\end{array}$ & $\begin{array}{l}\text { Study } \\
\text { design. }\end{array}$ & $\begin{array}{l}\text { Trauma field triage } \\
\text { guidelines. }\end{array}$ & $\begin{array}{l}\mathrm{n}=753 \\
\text { (patients) }\end{array}$ & $\begin{array}{l}\text { Diagnostic } \\
\text { accuracy. }\end{array}$ & $\begin{array}{l}\text { The guidelines sensitivity } \\
\text { was } 95.1 \% \text { and positive } \\
\text { predictive value was } 18.2 \% \text {. }\end{array}$ \\
\hline $\begin{array}{l}\text { Pointer } \\
2001^{48}\end{array}$ & $\begin{array}{l}\text { Prospective, } \\
\text { observational } \\
\text { study. }\end{array}$ & $\begin{array}{l}\text { Study } \\
\text { design. }\end{array}$ & Prehospital triage. & $\begin{array}{l}\mathrm{n}=1,180 \\
\text { (patients) }\end{array}$ & $\begin{array}{l}\text { Diagnostic } \\
\text { accuracy. }\end{array}$ & $\begin{array}{l}\text { Paramedics using written } \\
\text { guidelines fall short of } \\
\text { an acceptable level of } \\
\text { triage accuracy to } \\
\text { determine disposition of } \\
\text { patients in the field. }\end{array}$ \\
\hline $\begin{array}{l}\text { Prasanthi } \\
2008^{49}\end{array}$ & $\begin{array}{l}\text { Retrospective } \\
\text { observational } \\
\text { study. }\end{array}$ & $\begin{array}{l}\text { Study } \\
\text { design. }\end{array}$ & $\begin{array}{l}\text { Prehospital stroke tool } \\
\text { (Cincinnati Prehospital } \\
\text { Stroke Scale, CSS). }\end{array}$ & $\begin{array}{l}\mathrm{n}=477 \\
\text { (patients) }\end{array}$ & $\begin{array}{l}\text { Diagnostic } \\
\text { accuracy. }\end{array}$ & $\begin{array}{l}\text { Sensitivity of } 44 \% \text { and a } \\
\text { PPV of } 40 \% \text { for paramedics } \\
\text { using CSS. }\end{array}$ \\
\hline $\begin{array}{l}\text { Quain } \\
2008^{32}\end{array}$ & $\begin{array}{l}\text { Prospective } \\
\text { cohort study. } \\
\text { Before and after. }\end{array}$ & $\begin{array}{l}\text { Study } \\
\text { design. }\end{array}$ & $\begin{array}{l}\text { Prehospital stroke } \\
\text { assessment tool } \\
\text { (PAST). }\end{array}$ & $\begin{array}{l}\text { Intervention: } \\
\mathrm{n}=232 \\
\text { Retrospective } \\
\text { control group: } \\
\mathrm{n}=205\end{array}$ & $\begin{array}{l}\text { Diagnostic } \\
\text { accuracy. } \\
\text { Time to } \\
\text { definitive care. }\end{array}$ & $\begin{array}{l}\text { The proportion of } \\
\text { ischaemic stroke patients } \\
\text { treated with tPA } \\
\text { increased from } 4.7 \% \\
\text { (pre-intervention) to } \\
21.4 \% \text { (post-intervention) } \\
(p<0.001) \text {. Reduction in } \\
\text { median times from } \\
\text { symptom onset to ED } \\
\text { arrival from } 150 \text { to } \\
90.5 \text { min }(p=0,004) \text {. }\end{array}$ \\
\hline
\end{tabular}

Table 1 (continued) - Study characteristics of excluded studies of prehospital decision support 


\begin{tabular}{|c|c|c|c|c|c|c|}
\hline $\begin{array}{l}\text { Richman } \\
2008^{50}\end{array}$ & $\begin{array}{l}\text { Retrospective } \\
\text { observational } \\
\text { study. }\end{array}$ & $\begin{array}{l}\text { Study } \\
\text { design. }\end{array}$ & $\begin{array}{l}\text { Out-of-hospital } \\
\text { Termination of } \\
\text { Resuscitation (TOR) } \\
\text { Clinical Decision Rule. }\end{array}$ & $\begin{array}{l}\mathrm{n}=2,180 \\
\text { (patients) }\end{array}$ & $\begin{array}{l}\text { Diagnostic } \\
\text { accuracy. }\end{array}$ & $\begin{array}{l}\text { With respect to TOR, } \\
1,160 \text { of } 2,180(53.2 \%) \\
\text { patients met all three } \\
\text { criteria; only one }(0.09 \% \text {; } \\
95 \% \mathrm{Cl}=0 \% \text { to } 0.5 \%) \\
\text { survived to hospital } \\
\text { discharge. }\end{array}$ \\
\hline $\begin{array}{l}\text { Schmidt } \\
2000 \\
20016,\end{array}$ & $\begin{array}{l}\text { Prospective, } \\
\text { observational } \\
\text { study. }\end{array}$ & $\begin{array}{l}\text { Study } \\
\text { design. }\end{array}$ & $\begin{array}{l}\text { Treat and refer } \\
\text { protocol. }\end{array}$ & $\begin{array}{l}\mathrm{n}=1,300 \\
\text { (patients) }\end{array}$ & $\begin{array}{l}\text { Diagnostic } \\
\text { accuracy. }\end{array}$ & $\begin{array}{l}\text { From } 3 \% \text { to } 11 \% \text { of } \\
\text { patients determined on } \\
\text { scene not to need an } \\
\text { ambulance had a critical } \\
\text { event. Hospital } \\
\text { outcomes }=9 \% \\
\text { undertriage rate. }\end{array}$ \\
\hline $\begin{array}{l}\text { Snooks } \\
2004^{52}\end{array}$ & $\begin{array}{l}\text { Randomised } \\
\text { cohort study. }\end{array}$ & $\begin{array}{l}\text { Study } \\
\text { design. } \\
\text { SIGN } \\
50=-\end{array}$ & $\begin{array}{l}\text { Treat and refer } \\
\text { protocol. }\end{array}$ & $\begin{array}{l}\text { Intervention: } \\
n=251 \\
\text { Control group: } \\
n=537\end{array}$ & $\begin{array}{l}\text { Diagnostic } \\
\text { accuracy. }\end{array}$ & $\begin{array}{l}\text { "Treat and Refer" } \\
\text { protocols did not increase } \\
\text { the number of patients } \\
\text { left at home but were } \\
\text { used by crews and were } \\
\text { acceptable to patients. }\end{array}$ \\
\hline $\begin{array}{l}\text { Van't Hof } \\
2006^{53}\end{array}$ & $\begin{array}{l}\text { Non randomised } \\
\text { cohort study. }\end{array}$ & $\begin{array}{l}\text { Study } \\
\text { design. }\end{array}$ & $\begin{array}{l}\text { Computerized } \\
\text { electrocardiographic } \\
\text { algorithm. }\end{array}$ & $\begin{array}{l}\text { Ambulance } \\
\text { group, } n=209 \\
\text { Referred } \\
\text { group, } n=258\end{array}$ & $\begin{array}{l}\text { Diagnostic } \\
\text { accuracy. }\end{array}$ & $\begin{array}{l}\text { Acute myocardial } \\
\text { infarction was accurately } \\
\text { diagnosed in } 95 \% \text { of } \\
\text { patients in the ambulance } \\
\text { group as compared with } \\
99 \% \text { in the referred group } \\
(p=0.01) \text {. }\end{array}$ \\
\hline $\begin{array}{l}\text { Wojner- } \\
\text { Alexandrov } \\
2005^{33}\end{array}$ & $\begin{array}{l}\text { Prospective } \\
\text { cohort study. } \\
\text { Before and } \\
\text { after. }\end{array}$ & $\begin{array}{l}\text { Study } \\
\text { design. }\end{array}$ & $\begin{array}{l}\text { Los Angeles } \\
\text { Prehospital Stroke } \\
\text { Screen (LAPSS). }\end{array}$ & $\begin{array}{l}\text { Intervention: } \\
\mathrm{n}=446 \\
\text { Retrospective } \\
\text { control group: } \\
\mathrm{n}=1,072\end{array}$ & $\begin{array}{l}\text { Diagnostic } \\
\text { accuracy. }\end{array}$ & $\begin{array}{l}\text { Accuracy of paramedic } \\
\text { diagnosis of stroke } \\
\text { increased from } 61 \% \text { to } \\
79 \% \text {. Admission within } \\
2 \text { hours of symptom } \\
\text { onset increased from } \\
58 \% \text { to } 62 \%(p=0.002) \text {. }\end{array}$ \\
\hline
\end{tabular}

Table 1 (continued) - Study characteristics of excluded studies of prehospital decision support

thrombolysed $(p<0.05)$. The study also evaluated the number of confirmed strokes in the two groups. The control group had a higher proportion of confirmed strokes $(71.8 \%$ vs. $63.6 \%$ $(p<0.05)$. The results suggest that there is a high level of overdiagnosing in the intervention group and that could be a disadvantage with the use of a DST.

Time to Definitive Care-In a study with the aim to evaluate if a DST involving use of a continuous telemetry system and decision support by text message from a senior cardiologist could reduce the call-to-thrombolysis time. ${ }^{22}$ The median time in minutes (mins) from call to thrombolysis time was compared between patients receiving a recommendation for prehospital thrombolysis and controls thrombolysed in hospital. The median time in the intervention group was 53 minutes compared to 108 minutes in the control group. There was a difference of -55 minutes $(p<0.05)$. However, the control group had no possibilities to receive prehospital thrombolysis and the transport time to the hospital was an advantage for the intervention group. An interesting result was that the intervention group spent more "time on route" ( +10 minutes, $p<0.05)$, and according to "median call to door time" the control group was 19 minutes faster $(p<0.05)$, but "median door to thrombolysis time" favored the intervention group by -11 minutes. The difference was not statistically significant $(p=0.730)$, but it gives a hint about the DSTs impact on the time spent in hospital before definitive care.
In the ECP study, the outcome of interest was time from callto-hospital for EMS suspected stroke patients. ${ }^{21}$ This analysis used data from the "metropolitan area" where the distance to the hospital did not differ much. The intervention group spent four minutes less time during the transport $(p<0.05)$.

No study presented outcome data of compliance with recommended care, outcomes, patient or personnel satisfaction.

\section{Discussion}

A DST in prehospital care may have the possibility to decrease the "time to definitive care" and improve "diagnostic accuracy" provided by prehospital personnel. But more RCTs are needed.

It is important that only one of the included studies was aimed primarily at evaluating a DST. ${ }^{22}$ The ECP study included DSTs and this review selected data that supported the use of DSTs for prehospital decision making. ${ }^{21}$

The data from the included studies suggested that even if the use of a DST increases the prehospital time, it has the potential to decrease the time to definitive care. Factors that contribute to the latter could be that the prehospital assessment with a DST is more exhaustive in compared to assessment without support of a DST and the prehospital diagnosis more precise and this, in turn, may mean a shorter management in the hospital. However, there also is a risk that technical difficulties could increase the time spent outside the hospital. 


\begin{tabular}{|c|c|c|c|c|}
\hline $\begin{array}{l}\text { De Luca } \\
2009\end{array}$ & $\begin{array}{l}\text { EMS group } \\
\text { ITT analysis } \\
\text { Intervention: } \\
\mathrm{n}=1,181 \\
\text { Dropout: } \\
\mathrm{n}=291 \\
\text { Control: } \\
\mathrm{n}=720 \\
\text { Dropout: } \\
\mathrm{n}=41\end{array}$ & $\begin{array}{l}\text { Sign50 } \\
=++\end{array}$ & $\begin{array}{l}\text { The clinical pathway is based on available evidence } \\
\text { based prehospital and emergency interventions } \\
\text { for suspected stroke patients. It consists of the } \\
\text { following main points: } \\
\text { - Emergency service (ES) dispatcher uses a short } \\
\text { form of Cincinnati pre-Hospital Stroke Scale } \\
\text { (CHSS) to identify suspected stroke patients } \\
\text { during the telephone call; } \\
\text { - ES health workers confirm diagnosis by CHSS on } \\
\text { the scene; } \\
\text { - patients are provided CT and referred to } \\
\text { appropriate care. } \\
\text { A group of trainers from the expert panel that developed } \\
\text { the clinical pathway and composed of: } \\
\text { - an ES medical doctor - anaesthesiologist working } \\
\text { on helicopter emergency unit } \\
\text { - two neurologists working in stroke unit } \\
\text { - a physician working in an emergency department } \\
\text { - a neurosurgeon } \\
\text { - two epidemiologist and evidence-based medicine } \\
\text { expert trained a group of health workers selected } \\
\text { from all the entities participating in the intervention } \\
\text { arm study to act as "facilitators" for peer educa- } \\
\text { tion. The facilitators trained their colleagues in the } \\
\text { workplace with the help of audiovisual materials } \\
\text { produced by the teachers themselves. }\end{array}$ & $\begin{array}{l}\text { Of } 109 \text { eligible patients } 68 \text { patients } \\
(62.4 \%) \text { were referred to SU } \\
\text { compared to } 9 \text { of } 56(16.1 \%) \\
\text { eligible patients in the control } \\
\text { group (RR } 4.09 ; 95 \% \mathrm{Cl} \text { accounting } \\
\text { for clustering } 1.84 \text { to } 5.59 \\
\text { The intervention group spent } \\
\text { four minutes less time during the } \\
\text { transport }(p<0.05) \text {. }\end{array}$ \\
\hline $\begin{array}{l}\text { Woollard } \\
2005\end{array}$ & $\begin{array}{l}\text { Intervention: } \\
\mathrm{n}=213 \\
\text { Control: } \\
\mathrm{n}=183\end{array}$ & $\begin{array}{l}\text { Sign50 } \\
=+\end{array}$ & $\begin{array}{l}\text { Telemetry group patients received standard } \\
\text { pre-hospital treatment including direct admission to } \\
\text { the CCU plus continuous transmission of } 12 \text { lead } \\
\text { ECG, pulse oximetry and blood pressure while the } \\
\text { ambulance was on route to the hospital. Medical } \\
\text { history was also transmitted. A cardiology senior } \\
\text { house officer used this data to make } \\
\text { recommendations about suitability for prehospital } \\
\text { thrombolysis. Patients in the control group received } \\
\text { standard treatment. }\end{array}$ & $\begin{array}{l}\text { The median time in minutes from } \\
\text { call to thrombolysis time was } \\
\text { compared between patients } \\
\text { receiving recommendation for } \\
\text { prehospital thrombolysis and } \\
\text { controls thrombolysed in hospital. } \\
\text { The median time in the intervention } \\
\text { group was } 53 \text { minutes compared } \\
\text { to } 108 \text { minutes in the control group. } \\
\text { There was a difference of } \\
-55 \text { minutes }(p<0.05) \text {. }\end{array}$ \\
\hline
\end{tabular}

Table 2-Study characteristics and main findings of randomised controlled trials of prehospital decision support.

In some emergencies, the prehospital time can be crucial for the patient's outcome. For example, decreasing prehospital time matters in cases of serious trauma, ${ }^{54}$ acute ischemic heart disease, ${ }^{55}$ and stroke. ${ }^{56}$ Therefore, it is of importance that the DST has a large amount of usability.

None of the included studies evaluated the use of the DTS's impact on "compliance with recommended care". ${ }^{21,22}$ In-hospital care rates of compliance with evidence-based treatment guidelines commonly are used to evaluate quality of care. One study has demonstrated that compliance with guidelines improved outcomes for patients with acute coronary syndromes. ${ }^{20}$ There also are indications that patients with acute coronary syndromes who are transported in ambulances have a better outcome than do patients who arrive to emergency care in other ways. ${ }^{57}$ However, there are several studies that indicate that compliance with recommended care sometimes is poor in prehospital care. In a recent study where the compliance with recommended care for patients with chest pain who were treated by paramedics, was evaluated, the results showed the compliance with the comprehensive metric was poor. ${ }^{58}$ Other studies that compared prehospital treatment of men and women showed that women got less aspirin, nitroglycerin, and pain relief than did men. ${ }^{59}$ These findings are supported by additional studies. ${ }^{60,61}$ In addition, one study aimed to evaluate if paramedics could be helpful in determining if patients required emergency care showed paramedics failed to predict which patients do and do not require ED care. ${ }^{62}$

One of the main findings is the lack of RCTs in evaluation of DSTs in prehospital care. It is very surprising that many studies are well conducted but not randomized. Different kinds of DST for hospital and primary care have been well investigated with RCTs, Dexheimer included 61 RCTs in their systematic review over reminders in preventive care. ${ }^{64}$ In another systematic review, Kawamoto identified 88 eligible RCTs in an analysis of electronic and non-electronic decision system designed to aid directly in clinical decision. ${ }^{11}$ The review indicated that compliance with recommended care increased with the use of a DST particularly if the DST is computer-based and automatically gives decision support as part of clinician workflow. Shojania reviewed the effect of on screen reminders and included $28 \mathrm{RCT}$ s and quasi-randomized trials. ${ }^{14}$ Future research in prehospital decision support should concentrate on development and evaluation of computer-based decision support with the most important endpoint being patient outcome.

The excluded studies pinpointed areas of interest for the prehospital setting even if they didn't fulfill the criteria for RCT 
studies. Among those studies, nine evaluated the impact of different kinds of "stroke assessment tools". 23,29,32,33,35,40,43,45,4 9 Another frequent research issue among the excluded studies are treat and refer protocols (protocol and guidelines with the purpose to support prehospital personnel in the decision for level of care). ${ }^{37,38,42,51,52}$ Six studies evaluated prehospital triage tools. ${ }^{25,33,36,47,48,53}$ Interventions of prehospital cardiac care were evaluated in five studies, and three studies investigated the impact of prehospital 12-lead electrocardiograms (ECGs). 26,27,30 One study investigated prehospital AMI diagnosis, ${ }^{53}$ and a study evaluate protocol for percutaneous coronary intervention (PCI) bypass. ${ }^{30}$ The majority of these studies demonstrate a positive correlation between DST and impact on patient assessment.

\section{Potential Biases in the Review Process}

The search process was systematic. Studies that were missed in the medical database search were detected in the hand search of the 18 magazines. The search process involved searching of the "grey literature". In a systematic review with an aim to investigate the impact of the grey literature using meta-analyses, ${ }^{63}$ the result showed that published studies showed an overall greater treatment effect than grey trials. Searching for grey literature can be difficult and it is a risk that studies not published in medical literature have been missed in the search.

To evaluate the impact of DST on prehospital care, the researcher must be clear about what characterizes a DST. This review used Perreault and Metzger's definition as a guide, "Supporting clinical diagnosis and treatment plan processes; and promoting use of best practices, condition-specific guidelines, and population-based management." ${ }^{19}$ It still can be difficult to

References

1. Shaban R, Wyatt Smith CM, Cumming J: Uncertainty, error and risk in human clinical judgment: Introductory theoretical frameworks in paramedic practice. Journal of Emergency Primary Health Care 2004;2(1-2).

2. Brazier H, Murphy AW, Lynch C, Bury G: Searching for the evidence in prehospital care: A review of randomised controlled trials. On behalf of the Ambulance Response Time Sub-Group of the National Ambulance Advisory Committee. J Accid Emerg Med 1999;16(1):18-23.

3. Gunnarsson BM, Warrén Stomberg M: Factors influencing decision making among ambulance nurses in emergency care situations. Int Emerg Nurs 2009;17(2):83-89.

4. Wireklint Sundström B: Förberedd på att vara oförberedd: En fenomenologisk studie av vårdande bedömning och dess lärande $i$ ambulanssjukvård. 2005, Växjö: Växjö University Press.

5. Cooper S, Jr, Grant J: New and emerging roles in out of hospital emergency care: A review of the international literature. Int Emerg Nurs 2009;17(2):90-98.

6. Schmidt T, Atcherson K, Federiuk C, et al: Evaluation of protocols allowing emergency medical technicians to determine need for treatment and transport. Acad Emerg Med 2000;7(6):663-669.

7. Brown LH, Hubble MW, Cone DC, et al: Paramedic determinations of medical necessity: A meta-analysis. Prehosp Emerg Care 2009;13(4):516-527.

8. Rotter T, Kinsman L, James EL, et al: Clinical pathways: Effects on professional practice, patient outcomes, length of stay and hospital costs. Cochrane Database of Systematic Reviews, DOI: 10.1002/14651858.CD006632.pub2.

9. Snooks H, Evans A, Wells B, et al: What are the highest priorities for research in pre-hospital care? Results of a review and Delphi consultation exercise. Journal of Emergency Primary Health Care 2008;6(4):20.

10. Garg AX, Adhikari NK, McDonald H, et al: Effects of computerized clinical decision support systems on practitioner performance and patient outcomes: A systematic review. JAMA 2005;293(10):1223-1238.

11. Kawamoto K, Houlihan CA, Balas EA, Lobach DR: Improving clinical practice using clinical decision support systems: A systematic review of trials to identify features critical to success. BMJ 2005;330(7494):765-768.

12. Tan KRF, Dear P, Newell Simon J: Clinical decision support systems for neonatal care. Cochrane Database of Systematic Reviews, DOI: 10.1002/14651858. CD004211.pub2. determine the nature of a decision support. For example, in the PHT study the finally decision support to the prehospital personnel is given by a cardiologist at the nearby hospital via text message. ${ }^{22}$ It can be argued whether this is a DST. This review classified it as a DST because the cardiologist is part of a system involving 12-lead ECGs, study protocol and a thrombolysis eligibility questionnaire.

Another potential bias is difficulty in determining which factors in the intervention influenced the outcomes. When a prehospital organisation introduces a new DST, it usually is preceded by some sort of educational program. In the PHT study, the paramedic in the intervention group received 18 hours of training in 12-lead ECG recording, the telemetry equipment, the study protocol and the thrombolysis eligibility questionnaire. ${ }^{22}$ The personnel in the ECP study received a very thorough training performed in two steps. ${ }^{21}$ In the first step, interviews to identify the learning needs were conducted. In the second step, on-site training was conducted in small groups. The training focused on teaching the participants to identify stroke symptoms by the CSS. In these two studies, it was difficult to determine if it is the DST or the educational intervention that influenced the outcome and to what degree.

\section{Conclusions}

The dataset in this review is too small to be able to draw any reliable conclusions about the impact of DSTs on prehospital care. The search in this review indicates that there are very few RCTs that evaluate DSTs in prehospital care. Before different types of DSTs are introduced into the prehospital setting, the effects should be closely evaluated in well conducted RCTs.

13. Sintchenko V, Magrabi F, Tipper S: Are we measuring the right end-points? Variables that affect the impact of computerised decision support on patient outcomes: A systematic review. Medical Informatics $\mathcal{E}^{\circ}$ the Internet in Medicine 2007;32(3):225-240.

14. Shojania Kaveh G, Jennings A, Mayhew A, et al: The effects of on-screen, point of care computer reminders on processes and outcomes of care. Cochrane Database of Systematic Reviews DOI: 10.1002/14651858.CD001096.pub2; 2009.

15. Deeks J, Dinnes J, D'Amico R, et al: Evaluating non-randomised intervention studies. Health Technology Assessment 2003;7(27):1-173.

16. Scottish Intercollegiate Guidelines Network 2010 3/08/09.

17. Shukla VK, et al: Systematic review of quality assessment instruments for randomized control trials: Selection of SIGN50 methodological checklist [abstract]. XV Cochrane Colloquium; 2007 October 23-27; Sao Paulo, Brazil, 101.

18. Wyatt J, Spiegelhalter D: Field trials of medical decision-aids: Potential problems and solutions. in Proceedings/The Annual Symposium On Computer Application [Sic] In Medical Care. Symposium On Computer Applications In Medical Care. 1991.

19. Perreault LMJ: A pragmatic framework for understanding clinical decision support. Journal of Healthcare Information Management 1999;13(2):15-21.

20. Peterson ED, Roe MT, Mulgund J, et al: Association between hospital process performance and outcomes among patients with acute coronary syndromes. JAMA 2006;295(16):1912-1920.

21. De Luca A, Toni D, Lauria L, et al: (2009) An emergency clinical pathway for stroke patients-Results of a cluster randomised trial. BMC Health Services Research 2009;9(14).

22. Woollard M, Hayward AJ, Taylor NC: Limited benefits of ambulance telemetry in delivering early thrombolysis: A randomised controlled trial. Emerg Med J 2005;22(3):209-215.

23. Bray JE, Martin J, Cooper G, et al: An interventional study to improve paramedic diagnosis of stroke. Prehosp Emerg Care 2005;9(3):297-302.

24. Cayten CG, Oler J, Staroscik R, et al: Clinical algorithms for prehospital cardiac care. Medical Care 1983;21(2):147-156.

25. Ortolani P, Marzocchi A, Marrozzini C, et al: Usefulness of prehospital triage in patients with cardiogenic shock complicating ST-elevation myocardial infarction treated with primary percutaneous coronary intervention. Am J Cardiol 2007;100(5):787-792. 
26. Davis DP, Graydon C, Stein R, et al: The positive predictive value of paramedic versus emergency physician interpretation of the prehospital 12-lead electrocardiogram. Prehosp Emerg Care 2007;11(4):399-402.

27. Dhruva VN, Abdelhadi SI, Anis A, et al: ST-segment analysis using wireless technology in acute myocardial infarction (STAT-MI) trial. J Am Coll Cardiol 2007;50(6):509-513.

28. Watts D, Hanfling D, Waller M, et al: An evaluation of the use of guidelines in prehospital management of brain injury. Prehospital Emergency Care 2004;8(3): 254-261.

29. Gladstone DJ, Rodan LH, Sahlas DJ, et al: A citywide prehospital protocol increases access to stroke thrombolysis in toronto. Stroke 2009;40(12):3841-3844.

30. Le May MR, Davies RF, Dionne R, et al: Comparison of early mortality of paramedic-diagnosed ST-segment elevation myocardial infarction with immediate transport to a designated primary percutaneous coronary intervention center to that of similar patients transported to the nearest hospital. Am J Cardiol 2006;98(10):1329-1333.

31. Muhr MD, Seabrook DL, Wittwer LK: Paramedic use of a spinal injury clearance algorithm reduces spinal immobilization in the out-of-hospital setting. Prehosp Emerg Care 1999;3(1):1-6.

32. Quain DA, Parsons MW, Loudfoot AR, et al: Improving access to acute stroke therapies: A controlled trial of organised pre-hospital and emergency care. MedJ Aust 2008;189(8):429-433.

33. Wojner-Alexandrov AW, Alexandrov AV, Rodriguez D, et al: Houston Paramedic and Emergency Stroke Treatment and Outcomes Study (HoPSTO). Stroke 2005;36(7):15121518.

34. Burton JH, Harmon NR, Dunn MG, Bradshaw JR: EMS provider findings and interventions with a statewide EMS spine-assessment protocol. Prehosp Emerg Care 2005;9(3):303-309.

35. Kidwell CS, Saver JL, Schubert GB, et al: Design and retrospective analysis of the Los Angeles Prehospital Stroke Screen (LAPSS). Prehosp Emerg Care 1998;2(4):267-273.

36. Fries GR, McCalla G, Levitt MA, Cordova R: A prospective comparison of paramedic judgment and the trauma triage rule in the prehospital setting. Ann Emerg Med 1994;24(5):885-889.

37. Gray JT. Wardrope J: Introduction of non-transport guidelines into an ambulance service: a retrospective review. Emerg Med J2007;24(10):727-729.

38. Haines CJ, Lutes RE, Blaser M, Christopher NC: Paramedic initiated nontransport of pediatric patients. Prehosp Emerg Care 2006;10(2):213-219.

39. Jones T, Woollard M: Paramedic accuracy in using a decision support algorithm when recognising adult death: A prospective cohort study. Emerg Med J 2003;20(5):473-475.

40. Kidwell CS, Starkman S, Eckstein M, et al: Identifying stroke in the field : Prospective validation of the Los Angeles Prehospital Stroke Screen (LAPSS). Stroke 2000;31(1):71-76.

41. Klein KR, Spillane LL, Chiumento S, Schneider SM: Effects of on-line medical control in the prehospital treatment of atraumatic illness. Prehosp Emerg Care 1997;1(2):80-4.

42. Knapp BJ, Tsuchitani SN, Cheele JM, et al: Prospective evaluation of an emergency medical services-administered alternative transport protocol. Prehosp Emerg Care 2009;13(4):432-436.

43. Kothari RU, Pancioli A, Liu T, et al: Cincinnati Prehospital Stroke Scale: Reproducibility and validity. Ann Emerg Med 1999;33(4):373-378.

44. Meisel ZF, Mathew R, Wydro GC, et al: Multicenter validation of the Philadelphia EMS admission rule (PEAR) to predict hospital admission in adult patients using out-of-hospital data. Acad Emerg Med 2009;16(6):519-525.

45. Nor AM, McAllister C, Louw SJ, et al: Agreement between ambulance paramedicand physician-recorded neurological signs with Face Arm Speech Test (FAST) in acute stroke patients. Stroke 2004;35(6):355-1359.
46. Norcross ED, Ford DW, Cooper ME, et al: Application of American College of Surgeons' field triage guidelines by pre-hospital personnel. J Am Coll Surg 1995;181(6):539-544.

47. Paul AO, Kay MV, Huppertz T, et al: Validation of the prehospital mSTaRT triage algorithm. A pilot study for the development of a multicenter evaluation. Unfallchirurg 2009;112(1):23-30,32.

48. Pointer JE, Levitt MA, Young JC, et al: Can paramedics using guidelines accurately triage patients? Ann Emerg Med 2001;38(3):268-277.

49. Ramanujan P, Guluma KZ, Castillo EM, et al: Accuracy of stroke recognition by emergency medical dispatchers and paramedics-San Diego experience. Prehosp Emerg Care 2008;12(3):307-313.

50. Richman PB, Vadeboncoeur TF, Chikani V, et al: Independent evaluation of an out-of-hospital termination of resuscitation (TOR) clinical decision rule. Acad Emerg Med 2008;15(6):517-521.

51. Schmidt TA, Atcheson R, Federiuk C, et al: Hospital follow-up of patients categorized as not needing an ambulance using a set of emergency medical technician protocols. Prehosp Emerg Care 2001;5(4):366-370.

52. Snooks H, Kearsley N, Dale J, et al: Towards primary care for non-serious 999 callers: results of a controlled study of "Treat and Refer" protocols for ambulance crews. Qual Safe Health Care 2004;13(6):435-443.

53. van Hof AWJ, Rasoul S, van de Wetering H, et al: Feasibility and benefit of prehospital diagnosis, triage, and therapy by paramedics only in patients who are candidates for primary angioplasty for acute myocardial infarction. Am Heart $J$ 2006;151(6):1255.e1-5.

54. Gonzalez RP, Cummings GR, Phelan HA, et al: Does increased emergency medical services prehospital time affect patient mortality in rural motor vehicle crashes? A statewide analysis. Am J Surg 2009;197(1):30-34.

55. Boersma E, Maas E, Deckers JW, Simoons ML: Early thrombolytic treatment in acute myocardial infarction: reappraisal of the golden hour. Lancet 1996;348(9030):771-775.

56. Toni D, Chamorro A, Kaste M, et al: Acute treatment of ischaemic stroke. European Stroke Initiative. Cerebrovascular Diseases (Basel, Switzerland), 2004. 17 Suppl 2: p. 30-46.

57. Bettencourt N, Mateus P, Dias C, et al: Impact of pre-hospital emergency in the management and prognosis of acute myocardial infarction. Revista Portuguesa De Cardiologia: Orgiao Oficial Da Sociedade Portuguesa De Cardiologia 2005;24(6):863-872.

58. Colwell C, Mehler P, Harper J, et al: Measuring quality in the prehospital care of chest pain patients. Prehosp Emerg Care 2009;3(2):237-240.

59. Meisel ZF, Armstrong ZF, Mechem CC, et al: Influence of sex on the out-ofhospital management of chest pain. Acad Emerg Med 17(1):80-87.

60. Rittenberger JC, Beck PW, Paris PM: Errors of omission in the treatment of prehospital chest pain patients. Prehosp Emerg Care 2005;9(1):2-7.

61. Woollard M, Smith A, Elwood P: Pre-hospital aspirin for suspected myocardial infarction and acute coronary syndromes: a headache for paramedics? Emerg Med J 200;18(6):478-481.

62. Silvestri S, Rothrock SG, Kennedy D, et al: Can paramedics accurately identify patients who do not require emergency department care? Journal of Emergency Medicine 2002. 6(4): p. 387-390.

63. Hopewell S, McDonald S, Clarke M, Egger M: Grey literature in meta-analyses of randomized trials of health care interventions. Cochrane Database of Systematic Reviews DOI: 10.1002/14651858.MR000010.pub3.

64. Snooks H, Cheung W-Y, Close J, Dale J, Gaze S, Humphreys I, et al: Support and Assessment for Fall Emergency Referrals (SAFER 1) trial protocol. Computerised on-scene decision support for emergency ambulance staff to assess and plan care for older people who have fallen: evaluation of cost and benefits using a pragmatic cluster randomised trial. BMC Emergency Medicine. 2010;10:2. 